\title{
Pilot simulation of the temperature field of a continuous casting
}

\author{
J. Stetina ${ }^{1}$, F. Kavicka ${ }^{1}$, B. Sekanina ${ }^{1}$, J. Dobrovska ${ }^{2}$ \& J. Heger ${ }^{3}$ \\ ${ }^{I}$ Faculty of Mechanical Engineering, \\ Brno University of Technology, Czech Republic \\ ${ }^{2} V \check{S} B$ - Technical University of Ostrava, Czech Republic \\ ${ }^{3}$ ALSTOM Power Technology Centre, Leicester, U.K.
}

\begin{abstract}
Solidification and cooling of a continuously cast steel billet is a very complicated problem of transient heat and mass transfer. The solving of such a problem is impossible without a numerical model of the temperature field, not only of the concasting itself while it is being processed through the caster but of the mold as well. This process is described by the Fourier-Kirchhoff equation. An original 3D numerical off-line model of the temperature field of a caster has been developed. It has graphical input and output - automatic generation of the net and plotting of temperature fields in the form of color iso-therms and iso-zones, and temperature-time curves for any point of the system being investigated. This numerical model is capable of simulating the temperature field of a caster as a whole, or any of its parts. Experimental research and data acquisition have to be conducted simultaneously with the numerical computation-not only to confront it with the actual numerical model, but also to make it more accurate throughout the process. After computation, it is possible to obtain the temperatures at each node of the network, and at each time of the process. The utilization of the numerical model of solidification and cooling of a concasting plays an indispensable role in practice. The potential change of technology - on the basis of computation - is constantly guided by the effort to optimize, i.e. to maximize the quality of the process.
\end{abstract}

Keywords: concasting, solidification, temperature field, numerical model. 


\section{A numerical model}

Solidification and cooling of a casting and simultaneous heating of the mold is, from the point of view of heat transfer, a case of transient spatial, or 3D, heat and mass transfer in a system comprising the casting, mold and surroundings. This is a case of classical i.e. gravitational pouring. If mass transfer is not taken into account, and conduction is considered as the most important form of heat transfer, then the problem can be reduced to Fourier's partial differential equation.

$$
c_{v} \frac{\partial T}{\partial t}=\frac{\partial}{\partial x}\left(k \frac{\partial T}{\partial x}\right)+\frac{\partial}{\partial y}\left(k \frac{\partial T}{\partial x}\right)+\frac{\partial T}{\partial z}\left(k \frac{\partial T}{\partial z}\right)+Q_{\text {source }}
$$

In the case of continuous pouring, it is necessary to investigate the solidification and cooling of the concasting and the heating of the crystallizer. The 3D transient temperature field of a concasting, passing through a CCM (through the zones of primary, secondary, and tertiary cooling) in the direction of the z-axis at a shift rate of $w_{z}$, is described by the Fourier-Kirchhoff equation.

$$
c_{v}\left(\frac{\partial T}{\partial t}+w_{z} \frac{\partial T}{\partial z}\right)=\frac{\partial}{\partial x}\left(k \frac{\partial T}{\partial x}\right)+\frac{\partial}{\partial y}\left(k \frac{\partial T}{\partial x}\right)+\frac{\partial T}{\partial z}\left(k \frac{\partial T}{\partial z}\right)+Q_{\text {source }}
$$

In order to describe the temperature field of a concasting in the liquid phase, in the mushy zone, and in the solid phase, it is necessary to adapt this equation. In this case, it is necessary to introduce the specific volume enthalpy, which is dependent on temperature $\left(i_{v}=c_{v} . T\right)$. The specific volume heat capacity $c$ and conductivity $k$ are also functions of temperature. Equation (2) then transforms to

$$
\frac{\partial i_{v}}{\partial t}+w_{z} \frac{\partial I}{\partial z}=\frac{\partial}{\partial x}\left(k \frac{\partial T}{\partial x}\right)+\frac{\partial}{\partial y}\left(k \frac{\partial T}{\partial x}\right)+\frac{\partial T}{\partial z}\left(k \frac{\partial T}{\partial z}\right)
$$

The 3D transient temperature field of the crystallizer and frame, in which primary cooling takes place, is described by the Fourier equation (1) without the member $w_{z}(\partial T / \partial z)$. Figure 1 illustrates the thermal balance of an elementary volume (general nodal point $i, j, k$ ). The introduced unitary heat conductivities and heat flows in the directions of all main axes are indicated here too. The unitary heat conductivity in the direction of the $z$-axis is described by

$$
V Z_{i, j, k}=k(T) \frac{S_{z}}{\Delta z} \quad \text { or } \quad V Z_{i, j, k-1}=k(T) \frac{S_{z}}{\Delta z}
$$

The heat flows through the general nodal point $(i, j, k)$ in the $z$-direction are described by the following equations

$$
\begin{gathered}
Q Z 1_{i, j}=V Z_{i, j, k}\left(T_{i, j, k-1}^{(\tau)}-T_{i, j, k}^{(\tau)}\right) \\
Q Z_{i, j}=V Z_{i, j, k+1}\left(T_{i, j, k+1}^{(\tau)}-T_{i, j, k}^{(\tau)}\right)
\end{gathered}
$$


The temperature in the general nodal point of the concasting and the frame, in the course of a time step of $\Delta \tau$, is expressed by

$$
T_{i, j, k}^{(\tau+\Delta \tau)}=T_{i, j, k}^{(\tau)}+\left(Q Z 1_{i, j}+Q Z_{i, j}+Q Y 1_{i}+Q Y_{i}+Q X 1+Q X\right) \frac{\Delta t}{c_{v} \cdot \Delta x \cdot \Delta y \cdot \Delta z}
$$

The temperature in the general nodal point of the crystallizer in the course of the time step $(\Delta \tau)$ is determined from the specific volume enthalpy in time $\tau+\Delta \tau$, expressed

$$
i_{v_{i, j, k}}^{(\tau+\Delta \tau)}=i_{v_{i, j, k}}^{(\tau)}+\left(Q Z 1_{i, j}+Q Z_{i, j}+Q Y 1_{i}+Q Y_{i}+Q X 1+Q X\right) \frac{\Delta t}{\Delta x \cdot \Delta y \cdot \Delta z}
$$

The heat flow $Q Z_{i, j}$ in equation (8) is therefore given by the expression

$$
Q Z_{i, j}=V Z_{i, j, k}\left(T_{i, j, k+1}^{(\tau)}-T_{i, j, k}^{(\tau)}\right)-V Z_{i, j, k} \Delta z \cdot w_{z} \cdot i_{v_{i, j, k}}^{(\tau)}
$$

The accuracy of the presented numerical model depends not only on the spatial and temporal discretization $(\Delta \tau)$, but also on the accuracy with which the thermophysical properties of the material of all parts of the system are determined.
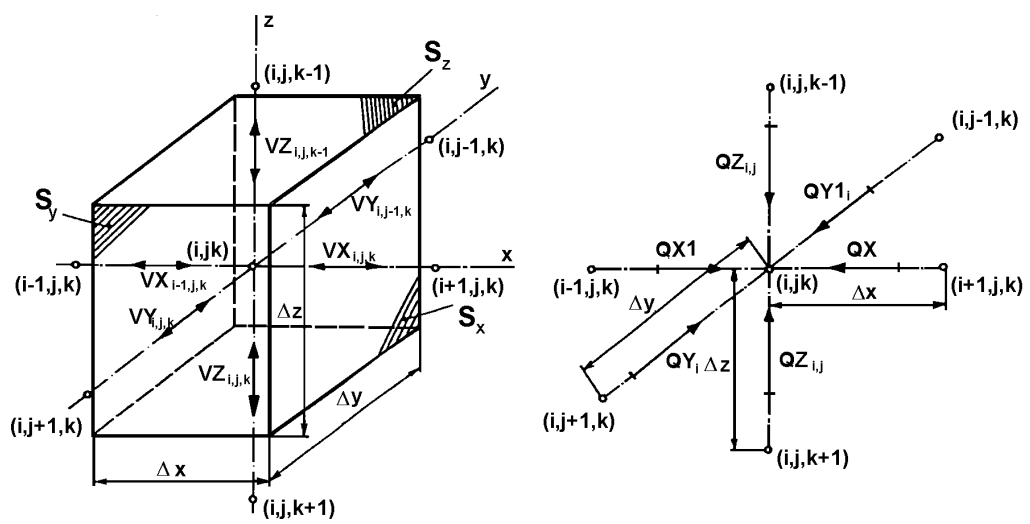

Figure 1: The thermal balance diagram of the general nodal point of the network.

The 3D model, outlined in this paper, is based on the explicit difference method. The numerical simulation of the release of latent heat of phase or structural changes is designed using the thermodynamic enthalpy function. It has a graphical input and output, i.e. automatic generation of the net (for an arbitrary shape of the crystallizer, and any profile of the concasting). The temperature fields are displayed in the form of color iso-therms, iso-zones and temperaturetime curves for any point of the system being investigated. 
An important part of this investigation is the measurement of the necessary quantities in the course of concasting. Experimental research enables acquisition of data in real time, which is necessary for optimization. This is ensured by the correct process procedure: real process $\rightarrow$ obtaining of input data $\rightarrow$ performing of the numerical analysis $\rightarrow$ optimization $\rightarrow$ correction of the real process. This procedure is necessary for optimization-especially when reacting to specific needs and conditions in the operation.

The program is designed to trigger the solving of sub-programs, which take over the systems generated by the pre-processing part of the program and, after completing the calculation, send files containing the results to the postprocessing part. The system developed is capable of performing all necessary tasks - from the generation of the net, through the determination of the thermophysical properties and the definition of boundary conditions, to the numerical simulation of the temperature field.

\section{The preparation for simulation}

The thermophysical properties of steels have significant influence on the actual concasting process, and on the accuracy of its numerical simulation and optimization. The determination of these properties often requires more time than the actual numerical calculation of the temperature fields of a continuously cast steel billet. The influence of individual properties should be neither undernor over-estimated. Therefore, an analysis/parametric study of these thermophysical properties was conducted. The order of importance within the actual process and the accuracy of simulation and optimization were also determined. Individual properties, which, in some cases, were obtained from tables, and in others experimentally, were substituted by an approximation using orthogonal polynomials. The accuracy of each polynomial is dependent on the precision of individual values.

The main properties are heat conductivity $\lambda$, density $\rho$ and specific heat capacity $c$, for the cast material in the liquid and in the solid state. These properties are dependent on temperature. The fourth property is latent heat of the phase change $L$. Latent heat of the steel phase change is about $2,67.10^{2}\left[\mathrm{~kJ} \cdot \mathrm{kg}^{-1}\right]$.

Furthermore, the exactness of the numerical model depends on the derivation of boundary conditions. Therefore the setting of the properties is followed by the setting of the boundary conditions, i.e. the values of the heat transfer coefficient (HTC) on all concasting machine (CCM) boundaries. The dependences of these coefficients on temperature and other operation parameters must also be given. The definition of boundary conditions is the most difficult part of the investigation of the thermokinetics of this process.

The boundary conditions of the numerical model of the temperature field of the concasting are defined as the heat transfer by convection (HTC). This HTC includes the so-called reduced convection coefficient corresponding to heat transfer by radiation.

The order of the importance of HTCs on each boundary has also been settled. In the system comprising the concasting and mould, the order is 
1. The $H T C$ at the point of contact between the concasting and mould (the influence of a cooling powder has also been included, see Figure 2).

2. The $H T C$ on the lower base of the mould.

3. The $H T C$ on the level of the melt and the upper base of the mould.

4. The $H T C$ on the outside wall of the mould.
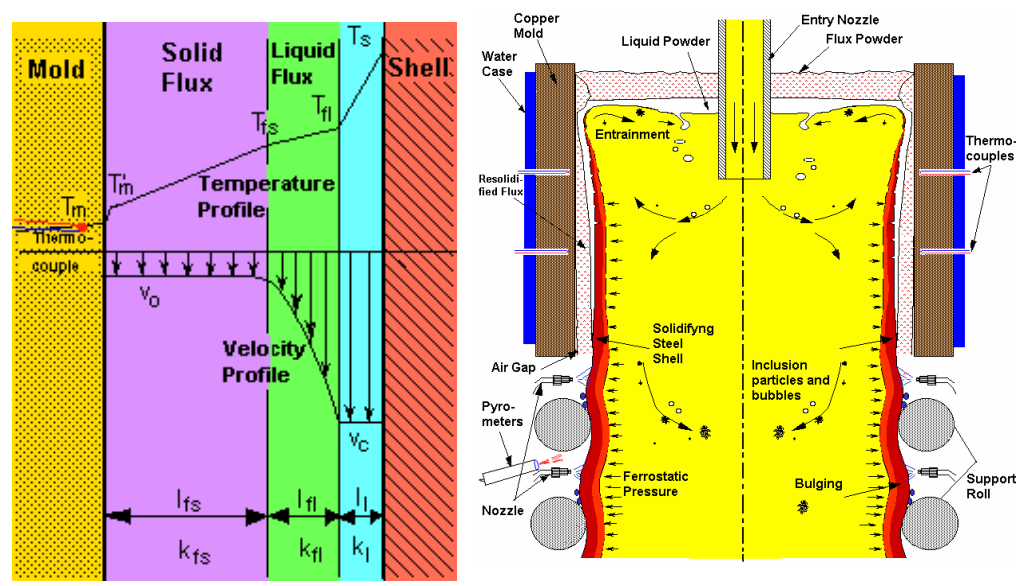

Figure 2: Model of heat transfer between shell and mold and arrangement of measurement sensors.

For example the boundary condition of the billet-mould interface depends on the thermophysical properties and the state of the casting powder, and also on the shape and size of the gap.

The only, but extremely important, coefficient, after leaving the mould, is the $H T C$ on the surface of the concasting, and is mainly dependent on the temperature of the surface, the shift rate and the intensity of spraying. This paper therefore continues with a discussion on heat transfer coefficients under cooling jets, which spray the concasting in the so-called secondary-cooling zone.

Regarding the fact that on a real CCM, where there are many types of jets with various settings positioned inside a closed cage, it is practically impossible to conduct measurement of the real boundary conditions. Therefore, a laboratory device was introduced in order to measure the cooling characteristics of the jets. This experimental laboratory device simulates not only the movement, but also the surface of a billet.

This laboratory device enables the measurement of each jet separately. It comprises a steel plate mounted with 18 thermocouples heated by an external electric source. The steel plate is heated to the testing temperature, than it is cooled by a cooling jet. On the return move the jet is covered by a deflector, which enables the movement of the jet without cooling the surface. This device measures the temperatures beneath the surface of the slab-again by means of thermocouples. 
The laboratory device allows the setting of:

- The jet type.

- The flow of water.

- The distance between the jet and the investigated surface.

- The surface temperature.

- The shift rate.

Based on the temperatures measured in dependence on time, the HTCs are calculated by an inverse task. They are then processed further using an expanded numerical and an identification model and converted to coefficients of the function $H T C(T, y, z)$ (Figure 3), which expresses the HTC in dependence on the surface temperature, and also the position of the concasting with respect to the jet.

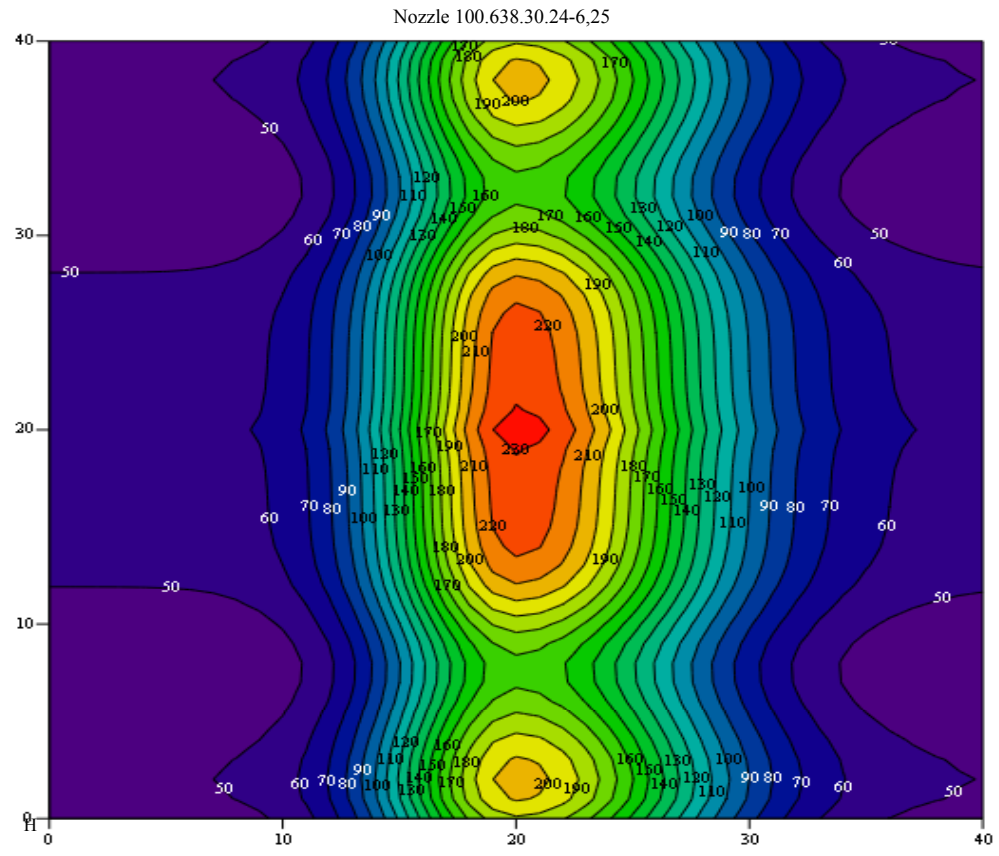

Figure 3: $\quad$ The HTC on the slab surface.

The experimental investigation is conducted also on an actual CCM during production. This investigation was focused mainly on the temperatures of the walls of the mould by means of thermocouples and on the surface temperatures of the slab under the mould by means of several pyrometers (see Figure 2).

\section{Application of the numerical model on a concast steel billet}

The numerical model described above is applied in order to investigate a concast steel billet with a square profile in any stage of the process. It has been decided 
to simulate the temperature field of a $150 \times 150 \mathrm{~mm}$ steel billet (30MnVS6). In order to determine the density of the mesh, the billet was analyzed in only one of its symmetrical halves, here on the right half. The selected density for the billet was 15 unit volumes in the x-direction, 30 unit volumes in the y-direction and 1000 unit volumes in the z-direction, which is the direction of a movement of the billet. The time step was selected within the range form 0.1 to $0.5 \mathrm{~s}$ and depends on the shift rate. A time step greater than $0.5 \mathrm{~s}$ does not guarantee numerically stable results. The temperature field of the billet was simulated for the shift rate 2 and $3 \mathrm{~m} / \mathrm{min}$.

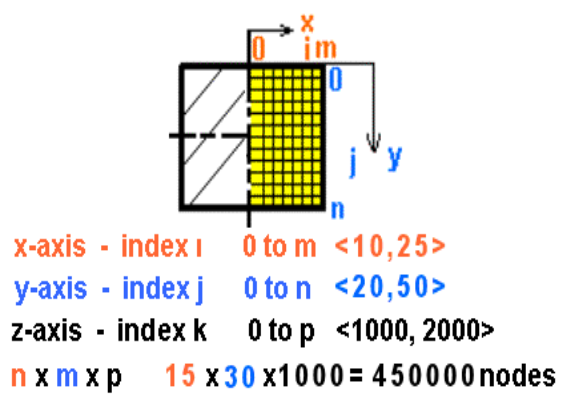

Figure 4: The billet square profile and the numerical mesh.

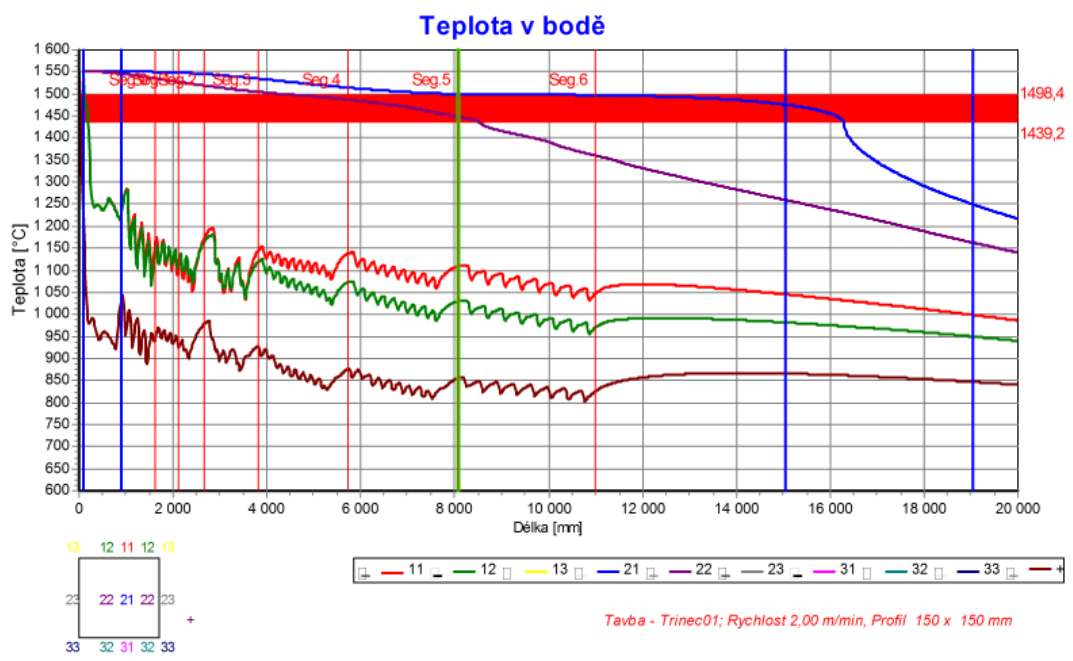

Figure 5: The temperature history in selected points of the cross-section of the billet (shift rate $2 \mathrm{~m} / \mathrm{min}$ ). 


\section{The results of a pilot calculation}

After the computation it is possible to obtain the temperatures at each node of the mesh and at any time of the process. Very useful are the 2D curves. Each curve shows the temperature history at user-defined point of the cross-section of the billet. Figure 5 represents these temperature-distance curves graphically for the shift rate $2 \mathrm{~m} / \mathrm{min}$ and Figure 6 for the shift rate $3 \mathrm{~m} / \mathrm{min}$.

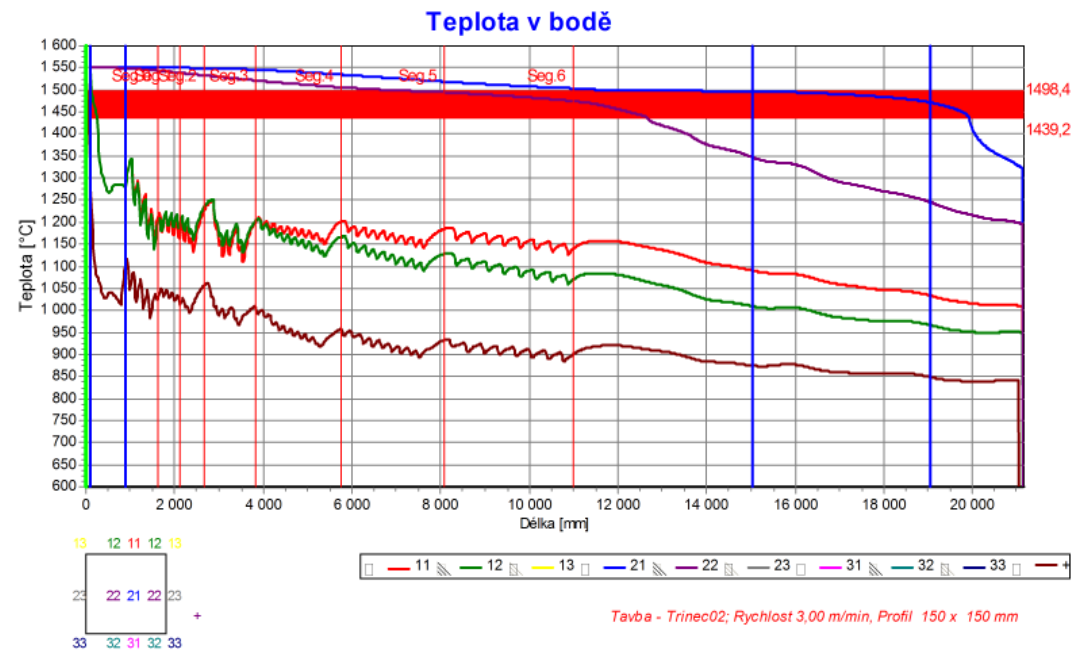

Figure 6: The temperature history in selected points of the cross-section of the billet (shift rate $3 \mathrm{~m} / \mathrm{min}$ ).

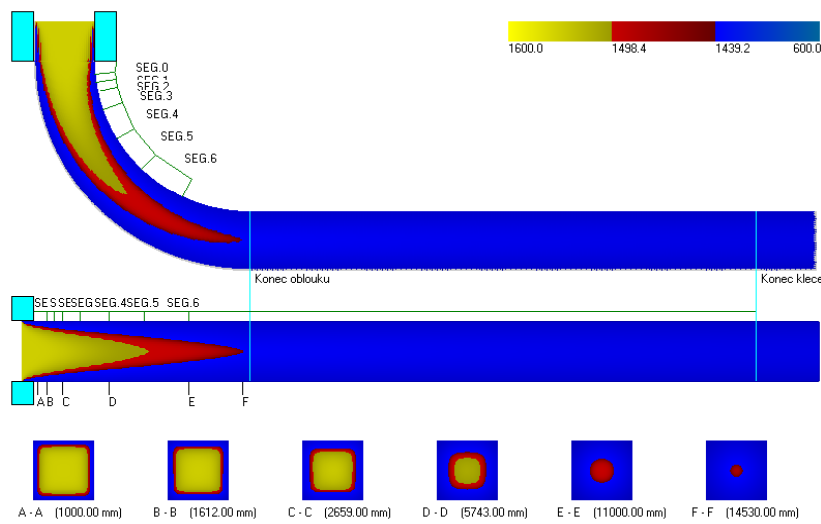

Figure 7: The temperature field of the longitudinal sections and cross sections of the billet (shift rate $2 \mathrm{~m} / \mathrm{min}$ ). 
The software allows the user to display or print the temperature field used isotherms or isozones or both whenever necessary. Figure 7 and 8 show the temperature field in the longitudinal sections of the billet $150 \times 150 \mathrm{~mm}$ for the shift rate $2 \mathrm{~m} / \mathrm{min}$ and $3 \mathrm{~m} / \mathrm{min}$. by means of isozones. Both figures represent also temperature isozones of the cross sections of the billet at different distances under the level in a mould. It is possible also to see a cone of solidification and its apex, i.e. metallurgical lengths.

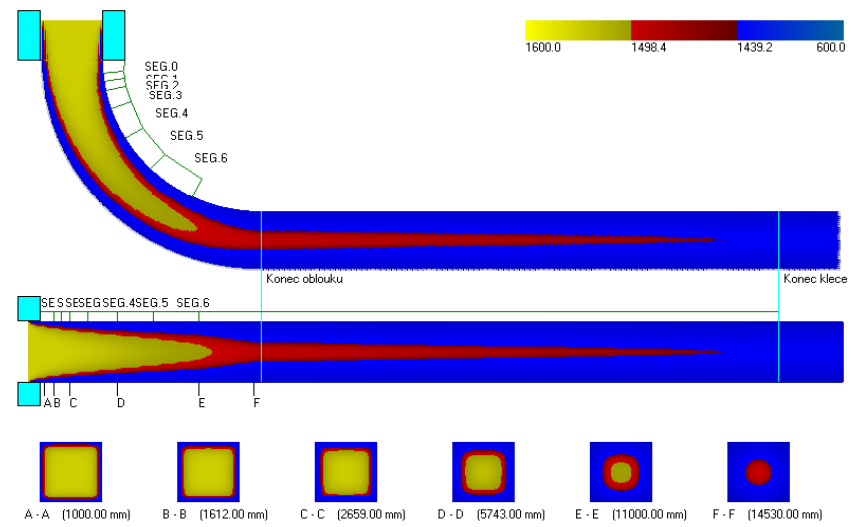

Figure 8: The temperature field of the longitudinal sections and cross sections of the billet (shift rate $3 \mathrm{~m} / \mathrm{min}$ ).

\section{Conclusion and outlook}

The presented model is a valuable computational tool and accurate simulator for investigating transient phenomena in billet-caster operations, and for developing control methods, the choice of an optimum cooling strategy to meet all quality requirements, and an assessment of the heat-energy content required for direct rolling. It should bring about quality improvement in the case of non-stable casting conditions. The on-line version will automatically set the technological parameters of the casting process in order to achieve the required quality of the cast steel.

\section{Acknowledgement}

This analysis was conducted using a program devised within the framework of the GA CR project No. 106/04/1334, 106/06/1210, 106/06/1225, CEZ MSM6198910013 and project MPO CR No. FI-IM/021. 


\section{Nomenclature}

\begin{tabular}{|c|c|c|}
\hline c & specific heat capacity & {$\left[\mathrm{J} \cdot \mathrm{kg}^{-1} \cdot \mathrm{K}^{-1}\right]$} \\
\hline$c_{v}$ & specific volume heat capacity $c_{v}=c . \rho$ & {$\left[\mathrm{J} \cdot \mathrm{m}^{-3} \cdot \mathrm{K}^{-1}\right]$} \\
\hline $\mathrm{h}$ & heat transfer coefficient & {$\left[\mathrm{W} \cdot \mathrm{m}^{-2} \cdot \mathrm{K}^{-1}\right]$} \\
\hline i & specific enthalpy & {$\left[\mathrm{J} . \mathrm{kg}^{-1}\right]$} \\
\hline $\mathrm{i}_{\mathrm{v}}$ & specific volume enthalpy $\quad i_{v}=i . \rho$ & {$\left[\mathrm{J} . \mathrm{m}^{-3}\right]$} \\
\hline $\mathrm{k}$ & heat conductivity & {$\left[\mathrm{W} \cdot \mathrm{m}^{-1} \cdot \mathrm{K}^{-1}\right]$} \\
\hline $\mathrm{w}_{\mathrm{z}}$ & shift rate & {$\left[\mathrm{m} \cdot \mathrm{s}^{-1}\right]$} \\
\hline $\mathrm{x}, \mathrm{y}, \mathrm{z}$ & axes in given directions & \\
\hline $\mathrm{Q}$ & heat flow in given direction & [W] \\
\hline QSOURCE & latent heat of the phase or structural change & {$\left[\mathrm{W} \cdot \mathrm{m}^{-3}\right]$} \\
\hline V & volume & {$\left[\mathrm{m}^{3}\right]$} \\
\hline $\mathrm{VX}, \mathrm{VY}, \mathrm{VZ}$ & unitary heat conductivity & {$\left[\mathrm{W} \cdot \mathrm{K}^{-1}\right]$} \\
\hline $\mathrm{T}$ & temperature & {$[\mathrm{K}]$} \\
\hline$\rho$ & density & {$\left[\mathrm{kg} \cdot \mathrm{m}^{-3}\right]$} \\
\hline$\tau$ & time & {$[\mathrm{s}]$} \\
\hline$\Delta \tau$ & time step & {$[\mathrm{s}]$} \\
\hline
\end{tabular}

\section{References}

[1] Miettinen J., Louhenkilpi \& Laine J., Solidification analysis package IDS. Proceeding of General COST 512 Workshop on Modelling in Materials Science and Processing, eds. M. Rappaz \& M. Kedro: ECSC-EC-EAEC, Brussels, Luxembourg, 1996.

[2] Thomas B.G., O’Malley R.J. \& Stone D.T., Measurement of temperature, solidification, and microstructure in a continuous cast thin slab. Paper presented at Modeling of Casting, Welding and Advanced Solidification Processes VIII: San Diego, CA, TMS, 1998.

[3] Brimacombe J. K., The Challenge of Quality in Continuous Casting Process. Metallurgical and Materials Trans. B, Volume 30B, pp. 553-566, 1999.

[4] Stetina J., Kavicka F., Dobrovska J., Camek L. \& Masarik M., Optimization of a concasting technology via a dynamic solidification model of a slab caster. Proceedings of the 5th Pacific International Conference on Advanced Materials and Processing, Peking, China, Part 5, p.3831-3834, 2004.

[5] Stetina J., Kavicka F., Dobrovska J., Camek L. \& Masarik M., Optimization of a concasting technology via a dynamic solidification model of a slab caster. Material Science Forum, Vol. 475-479, pp.3831-3834, Switzerland, 2005.

[6] Kavicka F., Stetina J., Stransky K., Sekanina B., Dobrovska J., Dobrovska V. \& Heger J., Optimization of a concasting technology by two numerical models. Proceedings of the ICAMT 2004 Third international conference on advanced manufacturing technology, Kuala Lumpur, Malaysia, pp. 647-653, 2004. 